\title{
Factors Associated with Death in Subjects Admitted for a Diabetological Emergency: Experience of the Medical Clinic II of the Abass Ndao Hospital Center in Dakar (Senegal)
}

\section{-Death in Diabetic Emergencies}

\author{
Diédhiou Demba1*, Sow Djiby¹, Diallo Ibrahima Mané1, Diallo Abdou Karim², \\ Ndour Michel Assane' ${ }^{1}$, Sarr Anna ${ }^{1}$, Ndour-Mbaye Maimouna ${ }^{1}$, Diop Said Norou ${ }^{1}$ \\ ${ }^{1}$ Department of Internal Medicine II, University Hospital Center of Dakar, Cheikh Anta Diop University, \\ Dakar, Senegal \\ ${ }^{2}$ Department of Preventive Medicine and Public Health, University Hospital Center of Dakar, Cheikh Anta Diop University, \\ Dakar, Senegal \\ Email: ^dembadiedhiou1976@gmail.com,drdjiby@yahoo.fr, ibrahimamanediallo@hotmail.fr, michelassanendour@yahoo.fr, \\ abdoulkarim7@outlook.com, mayoumbaye@gmail.com, annasarr@orange.sn, saidnorou@yahoo.fr
}

How to cite this paper: Demba, D., Djiby, S., Mané, D.I., Karim, D.A., Assane, N.M., Anna, S., Maimouna, N.-M. and Norou, D.S. (2018) Factors Associated with Death in Subjects Admitted for a Diabetological Emergency: Experience of the Medical Clinic II of the Abass Ndao Hospital Center in Dakar (Senegal). Open Journal of Endocrine and Metabolic Diseases, 8, 38-48. https://doi.org/10.4236/ojemd.2018.81005

Received: December 13, 2017

Accepted: January 19, 2018

Published: January 22, 2018

Copyright $(9) 2018$ by authors and Scientific Research Publishing Inc. This work is licensed under the Creative Commons Attribution International License (CC BY 4.0).

http://creativecommons.org/licenses/by/4.0/

\begin{abstract}
Introduction: Metabolic and vascular emergencies constitute a risk of lethality in diabetic subjects admitted to hospital. The objective was to evaluate the factors associated with death in subjects admitted for a diabetological emergency to improve management. Materials and Methods: It was a 12-month retrospective, descriptive and analytical study at Dakar's Marc Sankale Diabetes Center. The study concerned any type of diabetics subjects admitted for a diabetological emergency. We evaluated the profile of diabetes, the pathologies found and factors associated with death. Results: We identified 697 cases of diabetic emergencies with a prevalence of $13.8 \%$. The sex ratio $(\mathrm{M} / \mathrm{F})$ was 0.82 , the mean age was 49.6 years, and the mean duration of diabetes was 7.1 years. The metabolic profile was hypoglycemia (11.3\%), ketoacidosis (34.6\%), and hyperosmolar hyperglycemia syndrome (5\%). The associated pathologies were vascular (51.1\%), infectious (65.3\%), kidney function impairment (7.6\%), anaemia (13.8\%). During the follow-up, 94 patients died, as an annual frequency of $13.5 \%$ among diabetological emergencies. In univariate analysis, the factors significantly associated with death were age $>60$ years $[\mathrm{OR}=4.09(2.6$ - 6.41)], hyperglycemia [OR = $2.58(1.50$ - 4.4)], hyperosmolar hyperglycemia syndrome $[\mathrm{OR}=10.5(5.19-21.5)]$, septic diabetic foot $[\mathrm{OR}=3.57(2.24$ 5.66)]. Vascular pathologies that significantly associated with death were stroke
\end{abstract}


$[\mathrm{OR}=4.06(2.10-7.81)]$, lower limb arteriopathy $[\mathrm{OR}=3.25(1.84-5.73)]$, cardiovascular collapse [OR $=6.85(2.34-20)]$. In addition to diabetes, the deceased patients had one (18\%), two (34\%) and at least three (45.7\%) known factors of poor prognosis. Conclusion: Emergencies in diabetology remain frequent in our practice. The comorbidity (vascular and infectious) constitutes a risk of abnormally high death rate. A particular attention must concern on old diabetics subjects with several pathologies.

\section{Keywords}

Diabetological Emergencies, Mortality, Risk Factors, Senegal

\section{Introduction}

Diabetes mellitus is a public health problem with 425 million adults with diabetes mellitus worldwide. In Senegal, 3.24\% of the population were diabetic [1] [2]. Diabetes mellitus is a major cause of cardiovascular morbidity and mortality [3] [4]. In Africa, more than 298,160 deaths are reported to be related to diabetes and most of them among people are less than 60 years old [2]. The World Health Organization (WHO) predicts that by 2030, diabetes mellitus will be the seventh leading cause of death in the world [5]. The factors associated with death in diabetics subjects are unmodifiable like advanced age or in most cases related to acute (ketoacidosis and hyperosmolarity) and chronic complications of diabetes (arteritis). The latter constitutes the bulk of diabetological emergencies that are cardiovascular, metabolic and infectious [6] [7]. In sub-Saharan Africa, two series reported that metabolic (ketoacidosis and hyperosmolarity and hypoglycaemia) and vascular complications (stroke, arteriopathy, coronary heart disease) were the most factors associated with death in hospitalized diabetics [6] [8]. In Senegal, the few mortality studies in this population evaluated the isolated impact of metabolic and diabetics foot complications [9] [10]. These findings motivated our study, which aimed to make a global audit of the factors associated with death in subjects admitted to internal medicine for a diabetological emergency; thus to improve the care.

\section{Materials and Methods}

This was a retrospective, descriptive and analytical study conducted over 12 months (from January 1 to December 31, 2016). It was performed in the internal medicine department of the Medical Clinic II of the Abass Ndao University Hospital Center. Medical Clinic II included a department of internal medicine with diabetological orientation, a medical-surgical emergency unit, diagnostic assistance units and the Marc Sankale National Diabetes Center. The Marc Sankale Center has been the national reference center in the management of diabetes and metabolic diseases since 1960. It groups together therapeutic education, consultation and control units for the follow-up of known diabetics, diabetic foot care 
unit and finally a day admission unit of diabetological emergencies. Emergencies at night or on duty are transiently managed in the service of medical and surgical emergencies. The internal medicine unit is the traditional hospitalization unit for all cases in a persistent diabetological emergency, including severe hypoglycaemia; light to moderate forms are supported in day and guard emergency units.

The study included all cases of diabetic subjects admitted to Medical Clinical II during the study period for a diabetological emergency. We did not include the files of patients who died before admission, those considered incomplete, the files of diabetic subjects transferred to another hospital. For this survey, we selected the following variables:

- Sociodemographic Characteristics: sex, age.

- The Study of Diabetes Mellitus: type and duration of diabetes, level of glycemic balance, and associated cardiovascular risk factors known or discovered during hospitalization [11]. The known chronic complications of diabetes, antidiabetic therapies prior to hospitalization were also evaluated.

Pathologies Found during Hospitalization: these pathologies were retained after an overall evaluation of the data available in the file. These were constants such as weight $(\mathrm{kg})$, height $(\mathrm{m})$, body mass index $\left(\mathrm{kg} / \mathrm{m}^{2}\right)$, temperature $\left({ }^{\circ} \mathrm{C}\right)$, respiratory rate, diuresis, heart rate, blood pressure $(\mathrm{mmHg})$, glucosuria, ketonuria, and oxygen saturation. We also assessed the physical examination and exploration data. The recommendations of the Eighth Joint National Committee (JNC 8) were used to classify subjects according to blood pressure [12]. We also used the International Obesity Task Force's classification to classify patients according to the body mass index [13]. The electrocardiogram, the chest X-ray, the cytobacteriological study of the urine, the infectious assessments (hemogram, C-reactive protein), and kidney function evaluation were systematic in all the hospitalized patients. The other explorations were done according to the context. These included investigations of $\mathrm{pH}$, osmolarity, cultures and other bacteriological samples, vascular and imaging assessments. Metabolic disorders were defined according to international recommendations [14]. The ionic disorders accompanying the management of metabolic decompensation [15] were also evaluated. The main and secondary diagnoses were retained after a synthesis of the clinical and paraclinical data of the patients.

- The Hospital Outcome: we examined patient files to assess three possible outcomes: discharge from hospital, early transfer to another department and finally death during hospitalization. In a second step, we performed a comparative analysis between deceased and non-deceased patients in hospital to search for factors associated with death. The delay in death during hospitalization was also assessed. The parameters used in the evaluation of the factors associated with death were sociodemographic (age, sex), the profile of diabetes mellitus (type, duration, chronic complications), the type of diabetological emergency (infectious, metabolic, vascular), the others morbidities associated with 
diabetes mellitus observed during follow-up.

For the descriptive analysis, data were presented as a percentage for qualitative variables and on average for quantitative variables. The usual statistical tests used were the Chi-2 test for qualitative variables and the Student's test for quantitative variables. A $p$ value $<0.05$ was considered statistically significant with a 95\% confidence interval (CI). The recording and exploitation were carried out by SPSS STATISTICS 18.0 software.

\section{Results}

\subsection{Sociodemographic Data and Profile of Diabetes Mellitus}

During the study period, 5258 diabetic's subjects were recorded at Medical Clinic II. Among them, 697 were concerned by a diabetological emergency, as a prevalence of $13.3 \%$. The Table 1 show the epidemiological profile of all patients admitted for a diabetological emergency.

\subsection{Pathologies Found during Hospitalization}

- Glycemic Profile: all patients had a disturbance of the glycemic profile. Hypoglycemia was found in $11.3 \%$ (79 cases) with a mean age of 49.5 years, average diabetes duration of 9.9 years, including 17 cases of recent diabetes discovery. The ketoacidosis concerned 34.6\% (241 cases) with 75 cases of coma, a mean age of 43.5 years, average diabetes duration of 5.3 years including 62 cases of recent diabetes discovery. Diabetic hyperosmolarity syndrome was found in 5\% (35 cases) with 20 cases of coma, a mean age of 72.3 years, average diabetes duration of 7.1 years including 14 cases of recent

Table 1. Epidemiological profile of all patients admitted for a diabetological emergency.

\begin{tabular}{cc}
\hline Epidemiological Characteristics & \\
\hline Number of patients & 697 \\
Women & $382(54.8 \%)$ \\
Sex ratio (H/F) & 0.82 \\
Mean age of patients & 49.6 years \\
Patients $>60$ years old & $222(31.9 \%)$ \\
Patients $<30$ years old & $119(17.1 \%)$ \\
Type 1 diabetes & $197(28.3 \%)$, \\
Type 2 diabetes & $329(47.2 \%)$, \\
Recent-onset diabetes & $168(24.1 \%)$ \\
Mean duration of diabetes & 7.1 years \\
Duration of diabetes $\leq 5$ years & $363(52.1 \%)$ \\
Duration of diabetes $\geq 10$ years & $237(34.0 \%)$ \\
Oral antidiabetic agents & $238(34.1 \%)$ \\
Insulin treatment & $333(47.8 \%)$ \\
Diabetic mixed treatment & $9(01.3 \%)$ \\
\hline
\end{tabular}


diabetes discovery. A total of 95 patients (13.6\%) presented with hyperglycemic diabetic coma.

- Cardiovascular Profile: a vascular pathology was associated in 51.1\% (356 cases). It was hypertension in $38.5 \%$ (268 cases), lower limb arteriopathy in $10 \%$ (70 cases), stroke in $6.5 \%$ ( 5 cases), active coronary heart disease in $4.3 \%$ (30 cases), and hypokinetic cardiomyopathy with heart failure (2.9\%), heart rhythm or conduction disorders (1\%), and thromboembolic disease (1\%). In 14 cases (2\%), the patients were in cardiovascular collapse.

- Infectious Profile: an infection was found in 65.3\% (455 cases). It was a cutaneous infection in $25.4 \%$ (177 cases including 139 septic diabetic feet, 14 localizations at the hand and 24 abscesses), respiratory infection in $13.3 \%$ (93 cases including 15 cases of tuberculosis), and urogenital infection in 10.2\% (71 cases including 18 cases of pyelonephritis and 12 cases of prostatitis). Other locations were febrile gastroenteritis (2.4\%), bacterial meningoencephalitis $(0.9 \%)$, and cerebral malaria (6 cases, $0.9 \%)$. In 69 cases $(9.9 \%)$, this was an indeterminate sepsis.

- Other Associated Pathologies: renal function impairment was associated in $7.6 \%$ ( 53 cases) with a mean age of 56.6 years, an average duration of diabetes of 7.1 years, 7 cases of diabetes recently discovered. Among them, there were 2 cases of hypoglycaemia, 37 cases of ketosis and ketoacidosis and 11 cases of diabetic hyperosmolarity syndrome. Other morbidities were anaemia in 96 cases (13.8\%), cancer in 15 cases (2.2\%), and liver cirrhosis in 4 cases $(0.6 \%)$. The Table 2 shows the pathological profile of all patients admitted for a diabetological emergency.

\subsection{Outcome during Hospitalization and Profile of Deceased Patients}

The average duration of hospitalization was 8.3 days for all patients (range 1 to 60 days). Among the 697 subjects admitted for a diabetological emergency, 94 had died; as a frequency of $13.5 \%$. Their mean age was 61.9 years and $60.6 \%$ of patients were over 60 years old. The sex ratio $(\mathrm{M} / \mathrm{F})$ was 1.2 . The average duration of their diabetes was 7.9 years. In $44.6 \%$ of cases, the duration of diabetes was $\geq 10$ years. These included 13 cases of recent-onset diabetes, 7 cases of type 1 diabetes, and 74 cases of type 2 diabetes. The mean duration of hospitalization for deceased subjects was 4.5 days. In analyzing the records of deceased patients, the latter had, apart from diabetes mellitus, one factor (18\%), two factors (34\%), and three known factors (45.7\%) of poor prognosis. The most found pathologies were hyperglycemia and hyperosmolarity (45.7\%), ketosis and ketoacidosis (47.9\%), arterial hypertension (37.2\%), lower limb arteriopathy (20.2\%), stroke (17\%), infected diabetic foot (41.5\%), kidney function impairment (11.7\%) and anaemia (11.7\%). The Table 3 shows the pathological profile of the deceased patients.

\subsection{Factors Associated with Death}

Deceased patients were compared to non-deceased patients to assess the factors 
Table 2. Pathological profile of patients admitted for a diabetological emergency.

\begin{tabular}{lcc}
\hline Profile of diabetic emergencies $(\mathbf{n}=\mathbf{6 9 7})$ & Absolute frequency & Relative frequency \\
\hline Glycemic profile & 697 & $100 \%$ \\
Hypoglycemia & 79 & $11.3 \%$ \\
Ketosis and ketoacidosis & 494 & $70.9 \%$ \\
Hyperglycemia et hyperosmolarity & 124 & $17.8 \%$ \\
Cardiovascular profile & 356 & $51.1 \%$ \\
Arterial hypertension & 268 & $38.5 \%$ \\
Stroke & 45 & $06.5 \%$ \\
Acute coronary heart disease & 30 & $04.3 \%$ \\
Cardiomyopathy and collapsus & 34 & $04.9 \%$ \\
Lower limb arteriopathy & 70 & $10.0 \%$ \\
Infectious profile & 455 & $65.3 \%$ \\
Infected diabetic hand & 14 & $02.0 \%$ \\
Infected diabetic foot & 139 & $19.9 \%$ \\
Respiratory infection & 93 & $13.3 \%$ \\
Urogenital infection & 71 & $10.2 \%$ \\
Others infections & 134 & $19.2 \%$ \\
Others pathologies & & \\
Kidney function impairment & 53 & $07.6 \%$ \\
Anaemia & 96 & $13.8 \%$ \\
Cancer & 15 & $02.2 \%$ \\
Liver cirrhosis & 4 & $00.6 \%$ \\
\hline
\end{tabular}

Table 3. Pathological profile of deceased patients.

\begin{tabular}{lcc}
\hline \multicolumn{1}{c}{ Pathologies profile $(\mathbf{n}=\mathbf{9 4})$} & Absolute frequency & Relative frequency \\
\hline Glycemic abnormalities & 06 & $06.4 \%$ \\
Hypoglycemia & 43 & $45.7 \%$ \\
Hyperglycemia et hyperosmolarity & 45 & $47.9 \%$ \\
Ketosis and ketoacidosis & & \\
Infectious pathologies & 39 & $41.5 \%$ \\
Infected diabetic foot & 08 & $08.5 \%$ \\
Others infections & & \\
Cardiovascular pathologies & 35 & $37.2 \%$ \\
Arterial Hypertension & 16 & $17.0 \%$ \\
Stroke & 19 & $20.2 \%$ \\
Lower limb arteriopathy & 05 & $05.3 \%$ \\
Acute coronary heart disease & 04 & $04.3 \%$ \\
Cardiomyopathy in insufficiency & 07 & $07.4 \%$ \\
Cardiovascular collapsus & & $11.7 \%$ \\
Others pathologies & 11 & $11.7 \%$ \\
Kidney function impairment & 11 & $02.1 \%$ \\
Anaemia & 02 & \\
Cancer & &
\end{tabular}


associated with death. In univariate analysis, the most significantly associated factors with death were age $>60$ years $[(p<0.001)$, OR $=4.09(2.6-6.41)]$, hyperglycemia $[(p<0.001), \mathrm{OR}=2.58(1.50-4.4)]$, diabetic hyperosmolarity syndrome $[(p<0.001), \mathrm{OR}=10.5(5.19-21.5)]$. The infectious factors were not associated with death. In subgroup analysis, only the infected diabetic foot $[(p<$ $0.001), \mathrm{OR}=3.57(2.24-5.66)]$ was associated. The existence of an underlying cardiovascular pathology was a significant risk factor for death $(p<0.0001$. In subgroup analysis, the factors that were significantly associated with death were stroke $[(p<0.001), \mathrm{OR}=4.06(2.10-7.81)]$, lower limb arteriopathy $[(p<$ $0.0001, \mathrm{OR}=3.25(1.84-5.73)]$ and cardiovascular collapse $[(p=0.001), \mathrm{OR}=$ $6.85(2.34-20)]$. Table 4 shows the correlation between various factors relative to the deaths in patients admitted for diabetic emergencies.

\section{Discussion}

\subsection{Sociodemographic Data and Profile of Diabetes Mellitus}

The prevalence of diabetological emergencies is variable. Most studies address the subject according to a specific type of diabetological emergency [16] [17], or sometimes a more heterogeneous population involving both diabetic and non-diabetic subjects [6] [8] [18]. Chijioke et al. [8] in Nigeria reported a prevalence of 5.1\% in a population of type 2 diabetics among 15,320 admissions for all medical conditions combined. The socio-demographic profile of diabetic subjects, however, remains similar to data in the literature with a predominance of women, subjects mostly of advanced age and with type 2 diabetes most often formerly known [6] [8] [16] [17] [18].

\subsection{Pathologies Found during Hospitalization}

Diabetes emergencies are essentially metabolic, vascular and infectious [6] [7] [17]. In a 31 years retrospective study of a medical activity in Ghana, a predominance of metabolic forms was reported [6]. On the glycemic level, these were hyperglycemic emergencies (ketoacidosis and diabetic hyperosmolarity syndrome) in $29.8 \%$ and hypoglycaemic in $6.7 \%$ [8]. Our series, like most data in the literature, found infections and vascular pathologies respectively in second and third place as causes of diabetological emergencies [8] [19]. In these last two groups, the septic or mixed diabetic foot is a common and predominant reason for hospitalization. Kidney failure can be a factor of aggravation. In some studies, it is found in $17 \%$ [6] to $18 \%$ [19] of cases of diabetological emergencies.

\subsection{Profile of Deceased Patients}

The frequency of deaths following a diabetological emergency varies from $6.6 \%$ to $32 \%$ depending on the sample size, type of series and population studied. The same is true for the profile of deceased patients and the average duration of hospitalization [6] [7] [8] [19]. Zhu et al. [7], reported among 2,654 cases of death in diabetics admitted in emergency, a female predominance, a median age of 76 
Table 4. Factor associated with death among patients admitted in diabetological emergencies.

\begin{tabular}{|c|c|c|c|}
\hline \multirow{2}{*}{ Studied Parameters } & \multicolumn{2}{|c|}{ Dead Subjects } & \multirow{2}{*}{$p$ value and Odds Ratio (CI of $95 \%$ ) } \\
\hline & Yes & No & \\
\hline \multicolumn{4}{|l|}{ Gender } \\
\hline Men & $51(16.2 \%)$ & $264(83.8 \%)$ & \multirow[b]{2}{*}{$p=0.058$ and $\mathrm{OR}=0.65(0.40-1.01)$} \\
\hline Women & $43(11.3 \%)$ & $339(88.7 \%)$ & \\
\hline \multicolumn{4}{|l|}{ Age } \\
\hline$\leq 60$ years & $37(7.8 \%)$ & $438(92.2 \%)$ & \multirow{2}{*}{$p<0.001$ and $\mathrm{OR}=4.09(2.60-6.41)$} \\
\hline$>60$ years & $57(25.7 \%)$ & $165(74.3 \%)$ & \\
\hline \multicolumn{4}{|l|}{ Type of the diabetes mellitus } \\
\hline Type 1 & $07(3.6 \%)$ & $190(96.4 \%)$ & \multirow{2}{*}{$p<0.001$ and $\mathrm{OR}=0.12(0.06-0.28)$} \\
\hline Type 2 & $74(22.5 \%)$ & $255(77.5 \%)$ & \\
\hline \multicolumn{4}{|l|}{ Duration of the diabetes mellitus } \\
\hline$<10$ years & $52(11.3 \%)$ & $408(88.7 \%)$ & \multirow{2}{*}{$p=0.837$ and $\mathrm{OR}=0.94(0.55-1.60)$} \\
\hline$\geq 10$ years & $42(17.7 \%)$ & $195(82.3 \%)$ & \\
\hline \multicolumn{4}{|l|}{ Glycemic imbalance } \\
\hline Hypoglycemia & $06(7.6 \%)$ & $73(92.4 \%)$ & $p=0.110$ and $\mathrm{OR}=0.48(0.20-1.14)$ \\
\hline Hyperglycemia & $23(25.9 \%)$ & $66(74.1 \%)$ & $p<0.001$ and $\mathrm{OR}=2.58(1.50-4.40)$ \\
\hline Ketosis & $18(07.1 \%)$ & $235(92.9 \%)$ & $p<0.001$ and $\mathrm{OR}=0.37(0.21-0.63)$ \\
\hline Ketoacidosis & $27(11.2 \%)$ & $214(88.8 \%)$ & $p=0.240$ and $\mathrm{OR}=0.73(0.45-1.18)$ \\
\hline Hyperosmolarity & $20(57.1 \%)$ & $15(42.9 \%)$ & $p<0.001$ and $\mathrm{OR}=10.5(5.19-21.5)$ \\
\hline \multicolumn{4}{|l|}{ Others metabolic disorders } \\
\hline Anaemia & $15(15.6 \%)$ & $81(84.4 \%)$ & $p=0.520$ and $\mathrm{OR}=1.22(0.67-1.22)$ \\
\hline Renal failure \& ionic disorders & $10(18.9 \%)$ & $43(81.1 \%)$ & $p=0.210$ and $\mathrm{OR}=1.55(0.75-3.13)$ \\
\hline \multicolumn{4}{|l|}{ Infectious diseases } \\
\hline Infected diabetic foot & $39(28.1 \%)$ & $100(71.9 \%)$ & $p<0.001$ and $\mathrm{OR}=3.57(2.24-5.66)$ \\
\hline Respiratory infections & $04(4.3 \%)$ & $89(95.7 \%)$ & $p=0.080$ and $\mathrm{OR}=0.40(0.14-1.13)$ \\
\hline Urogenital infections & $02(2.8 \%)$ & $69(97.2 \%)$ & $p=0.003$ and $\mathrm{OR}=0.17(0.04-0.70)$ \\
\hline \multicolumn{4}{|l|}{ Cardiovascular diseases } \\
\hline Arterial hypertension & $35(13.1 \%)$ & $233(86.9 \%)$ & $p=0.820$ and $\mathrm{OR}=0.94(0.60-1.47)$ \\
\hline Stroke & $16(35.6 \%)$ & $29(64.4 \%)$ & $p<0.001$ and $\mathrm{OR}=4.06(2.10-7.81)$ \\
\hline Acute coronary heart disease & $05(16.7 \%)$ & $25(83.3 \%)$ & $p=0.570$ and $\mathrm{OR}=1.41(0.52-3.82)$ \\
\hline Lower limb arteriopathy & $19(27.1 \%)$ & $51(72.9 \%)$ & $p<0.001$ and $\mathrm{OR}=3.25(1.84-5.73)$ \\
\hline Hypokinetic cardiomyopathy & $04(20 \%)$ & $15(60 \%)$ & $p=0.720$ and $\mathrm{OR}=1.29(0.36-4.55)$ \\
\hline Collapse & $07(50 \%)$ & $7(50 \%)$ & $p=0.001$ and $\mathrm{OR}=6.85(2.34-20.0)$ \\
\hline Neoplasm & $02(10.5 \%)$ & $17(89.5 \%)$ & $p=1.000$ and $\mathrm{OR}=0.74(0.17-3.30)$ \\
\hline
\end{tabular}

Abbreviation: $\mathrm{OR}=$ Odds Ratio; $p=\mathrm{p}$ value; $\mathrm{CI}=$ Confidence Interval.

years, $44 \%$ of type 2 diabetes and $55.1 \%$ of cases of diabetes not yet classified. Our study also reveals a high morbidity of the deceased patients since they had, in addition to diabetes mellitus, 2 factors (34\%) and $\geq 3$ factors $(45.7 \%)$ of poor prognosis. 


\subsection{Factors Associated with Death}

In our study and like that of Sarfo-Kantanka et al. [6], the non-modifiable factors associated with death were older age, male sex and type 2 diabetes. In another Nigerian study, females were more likely to be incriminated [20]. Regarding to the type of diabetes, previous ignorance of diabetes mellitus seems to worsen the prognosis [7].

Metabolic complications are known to be among the most common poor prognostic factors in diabetic patients [7] [20]. They would account for $28.7 \%$ of death in a study of Zhu et al. [7] in China. Some data in the literature specifically criminalized ketoacidosis and hyperosmolar hyperglycemia syndrome [8] [19]. The negative effect of hypoglycemia was reported by other authors [6]. We report, however, a protective effect of ketosis $[(p=0.000$ and $\mathrm{OR}=0.37(0.21$ $0.63)]$ and diabetic ketoacidosis $[(p=0.24$ and $\mathrm{OR}=0.73(0.45-1.18)]$. These same findings were reported in a prospective cohort study [21], which compared 486 ketoacidosis with 486 non-ketoacidosis hyperglycaemic episodes. In multivariate analysis, diabetic ketoacidosis would be a mark of protection against lethality in subjects admitted in emergency $[(p=0.0005$ and $\mathrm{HR}=0.63(0.48$ $0.82)]$.

The causes of death in diabetic subjects also depend on the level of health transition. Infection does not appear to be a significant factor in poor prognosis [7]. A significant deleterious effect of respiratory infections associated with diabetic emergencies was reported by Zelada et al. [19]. In subgroup analysis, the negative impact of the infected or mixed diabetic foot remains unanimously recognized [6] [8]. In a study conducted in the United State of America, the diabetic foot is a major public health problem because of its frequency (1.9\% among 54.2 million diabetics), its morbidity (9.6\% risk of sepsis and $10.5 \%$ amputation) and its risk of mortality estimated to $2 \%$ [17]. Apart from underlying arteritis, sepsis is a factor of excess mortality in the cases of diabetic foot in 5.6\% [22].

Although infectious factors are still considerable in Sub-Saharan Africa, it is none the less true for vascular causes. They are found in $28.3 \%$ of our deceased patients. In a study in the United Kingdom, among the 9.8\% cause of death in type 2 diabetics, cardiovascular factors were reported in 3\% [23]. These were mainly stroke [7] [8] [19] and to a lesser extent coronary heart disease and lower limb arteriopathy [6].

\section{Limitations of the Study}

1) The study was conducted in university hospital, it may not represent the community situation which is important in terms of knowledge and practice.

2) This is a single center study. Large scale multicenter study is needed to represent the national situation.

3) This is a retrospective study conducted from patient's files. Only the complete files were included, which makes a loss of data. In more, retrospective character of this work was not able to highlight the impact in term of mortality of 
the coverage and of his deadline.

4) This kind of work doesn't require in our country (Senegal) the approval of ethic committee because it is a retrospective work on patient's file.

\section{Conclusion}

In our practice of diabetes, emergencies remain frequent. The metabolic forms remain preponderant. It is in fact a tripod in which certain infectious and vascular morbidities constitute an over-risk of mortality. In this context, the septic or mixed diabetic foot remains a major concern. Special attention should be paid to elderly diabetic subjects with several pathologies.

\section{Conflict of Interest}

The authors have nothing to disclose.

\section{References}

[1] Diop, S.N. and Diédhiou, D. (2015) Diabetes Mellitus in Sub-Saharan Africa: Epidemiological and Socioeconomic Aspects. Médecine des Maladies Métaboliques, 9 , 123-129. https://doi.org/10.1016/S1957-2557(15)30030-4

[2] International Diabetes Federation (IDF) (2017) IDF Diabetes Atlas. 8th Edition, IDF, Brussels.

[3] WHO (2016) World Report on the Diabetes Mellitus. World Health Organization, Geneva.

[4] Diedhiou, D., Sow, D., Diallo, I.M., Diouara, A., Ndour, M.A., Ndour-Mbaye, M., et al. (2017) Decentralized Management of Diabetes Mellitus by General Patrician. Open Journal of Internal Medicine, 7, 25-35. https://doi.org/10.4236/ojim.2017.72003

[5] Mathers, C.D. and Loncar, D. (2006) Projections of Global Mortality and Burden of Disease from 2002 to 2030. PLOS Medicine, 3, e442. https://doi.org/10.1371/journal.pmed.0030442

[6] Sarfo-Kantanka, O., Sarfo, F.S., Oparebea, A.E., Eghan, B., Ayisi-Boateng, N.K. and Acheamfour-Akowuah, E. (2016) Secular Trends in Admissions and Mortality Rates from Diabetes Mellitus in the Central Belt of Ghana: A 31-Year Review. PLoS ONE, 11, e0165905. https://doi.org/10.1371/journal.pone.0165905

[7] Meiying, Z., Jiang, L., Zhiyuan, L., Wei, L., Dajun, D., Weaver, S.R., et al. (2015) Mortality Rates and the Causes of Death Related to Diabetes Mellitus in Shanghai Songjiang District: An 11-Year Retrospective Analysis of Death Certificates. BMC Endocrine Disorders, 15, 45. https://doi.org/10.1186/s12902-015-0042-1

[8] Chijioke, A., Adamu, A.N. and Makusidi, A.M. (2010) Mortality Patterns among Type 2 Diabetes Mellitus Patients in Ilorin, Nigeria. JEMDSA, 15, 79-82. https://doi.org/10.1080/22201009.2010.10872231

[9] Diédhiou, D., Sarr, A., Leye, M.M.M., Ndour-Mbaye, M., Diouf, S.M., Ka-Cisse, M., et al. (2012) Foot Ulcer in Diabetes Mellitus: Epidemiological, Clinical, Etiological and Evolutional Aspects in Abass Ndao Hospital Center. Dakar Médical, 57, 154-161.

[10] Sarr, A., Diédhiou, D., Ndour-Mbaye, N.M., Leye, Y.M., Ka-Cisse, M.S., Leye, A., et al. (2011) Ketoacidosis in Type 1 Diabetes Mellitus: About 73 Cases in Dakar. Mali Medical, XXVI, 50-54. 
[11] AFSSAPS, High Authority of Health (2006) Medicinal Treatment of the Type 2 Diabetes Mellitus. Recommendations.

[12] James, P.A., Oparil, S., Carter, B.L., Pharm, B. et al. (2014) Evidence-Based Guideline for the Management of High Blood Pressure in Adults: Report from the Panel Members Appointed to the Eighth Joint National Committee (JNC 8). JAMA, 311, 507-520. https://doi.org/10.1001/jama.2013.284427

[13] Cole, T.J., Bellizzi, M.C., Flegal, K.M. and Diet, W.H. (2000) Establishing a Standard Definition for Overweight and Obesity Worldwide: International Survey. $B M J$, 320, 1240-1243. https://doi.org/10.1136/bmj.320.7244.1240

[14] Orban, C. and Ichai, C. (2008) Acute Metabolic Complications of Diabetes Mellitus. Réanimation, 17, 761-767. https://doi.org/10.1016/j.reaurg.2008.09.006

[15] Arora, S., Cheng, D., Wyler, B. and Menchine, M. (2012) Prevalence of Hypokalemia in ED Patients with Diabetic Ketoacidosis. American Journal of Emergency Medicine, 30, 481-484. https://doi.org/10.1016/j.ajem.2011.01.002

[16] Ezeani, I.U., Eregie, A. and Ogedengbe O.S. (2013) Treatment Outcome and Prognostic Indices in Patients with Hyperglycemic Emergencies. Diabetes, Metabolic Syndrome and Obesity: Targets and Therapy, 6, 303-307. https://doi.org/10.2147/DMSO.S44477

[17] Skrepnek, G.H., Mills, J.L. and Armstrong D.G. (2015) A Diabetic Emergency One Million Feet Long: Disparities and Burdens of Illness among Diabetic Foot Ulcer Cases within Emergency Departments in the United States, 2006-2010. PLoS ONE, 10, e0134914. https://doi.org/10.1371/journal.pone.0134914

[18] Ford, W., Self, W.H., Slovis, C. and McNaughton C.D. (2013) Diabetes in the Emergency Department and Hospital: Acute Care of Diabetes Patients. Current Emergency and Hospital Medicine Reports, 1, 1-9. https://doi.org/10.1007/s40138-012-0007-x

[19] Zelada, H., Bernabe-Ortiz, A. and Manrique H. (2016) Inhospital Mortality in Patients with Type 2 Diabetes Mellitus: A Prospective Cohort Study in Lima, Peru. Journal of Diabetes Research, 2016, Article ID 7287215. https://doi.org/10.1155/2016/7287215

[20] Ojobi, J.E., Odoh, G., Aniekwensi, E. and Dunga J. (2016) Mortality among Type 2 Diabetic In-Patients in a Nigerian Tertiary Hospital. African Journal of Diabetes Medicine, 24, 17-20.

[21] Kruljac, I., Ćaćić, M., Ćaćić, P., Ostojić, V., Štefanović, M., Šikić, A., et al. (2017) Diabetic Ketosis during Hyperglycemic Crisis Is Associated with Decreased All-Cause Mortality in Patients with Type 2 Diabetes Mellitus. Endocrine, 55, 139-143. https://doi.org/10.1007/s12020-016-1082-7

[22] Hadadi, A., Omdeh Ghiasi, H., Hajabdolbaghi, M., Zandekarimi, M. and Hamidian R. (2014) Diabetic Foot: Infections and Outcomes in Iranian Admitted Patients. Jundishapur Journal of Microbiology, 7, Article ID e11680. https://doi.org/10.5812/jjm.11680

[23] Taylor, K.S., Heneghan, C.J. and Farmeretal A.J. (2013) All-Cause and Cardiovascular Mortality in Middle-Aged People with Type 2 Diabetes Compared with People without Diabetes in a Large U.K. Primary Care Database. Diabetes Care, 36, 2366-2371. https://doi.org/10.2337/dc12-1513 